\title{
Revisión de la historia y aplicación de escuaramida en organocatálisis
}

\author{
Review of the history and application of squaramide in organocatalysis
}

Revisão da história e aplicação de escuaramida em organocatálise

\author{
Eduardo Hipólito Hernández ${ }^{1}$ \\ Nancy Romero-Ceronio ${ }^{2}$ \\ Cuauhtémoc Alvarado-Sánchez ${ }^{3}$ \\ Carlos E. Lobato-García ${ }^{4}$ \\ Miguel A. Vilchis-Reyes ${ }^{5}$ \\ Luis F. Roa ${ }^{6}$
}

Recibido: agosto de 2017

Aceptado: abril de 2018

Para citar este artículo: Hernández, E. H., Romero-Ceronio, N, Alvarado-Sánchez, C., Lobato-García, C. E., Vilchis-Reye, M. A., y Roa, L, F. (2018). Revisión de la historia y aplicación de escuaramida en organocatálisis. Revista Científica, 32(2), 135-146. Doi: https://doi.org/10.14483/23448350.12554

\section{Resumen}

La escuaramida y sus derivados representan un importante grupo de sustancias que actualmente se están aplicando exitosamente en estrategias de organocatálisis asimétrica. En esta contribución se presenta un resumen del estado del arte relativo a la síntesis de los derivados de escuaramida y su papel como inductores de quiralidad en secuencias sintéticas relevantes. Por ello, se establece un proceso de búsqueda sistemática y selección de reportes publicados en revistas especializadas del área, con la finalidad de presentar los aspectos más notables de síntesis y la aplicación de los derivados de escuaramida en esta sustancial área de la química orgánica. Palabras clave: química orgánica, síntesis asimétrica, organocatalizadores, inducción quiral, derivados de escuaramida.

\begin{abstract}
Squaramide and its derivatives represent an important group of substances that are currently being successfully applied in asymmetric organocatalitic strategies, which is why this contribution presents a summary of the state of the art related to the synthesis of escuaramide derivatives and their role as chirality inducers in relevant synthetic sequences. To this end, a process of systematic search and selection of reports published in specialized journals of the area was established in order to present the most significant aspects of synthesis and the application of escuaramide derivatives in this key area of organic chemistry.
\end{abstract}

Keywords: organic chemistry, asymmetric synthesis, organocatalysts, chiral induction, escuaramide derivatives.

\footnotetext{
Universidad Juárez Autónoma de Tabasco (UJAT), México. q.e.hipolito@gmail.com Universidad Juárez Autónoma de Tabasco (UJAT), México. nancy.romero@ujat.mx Universidad Juárez Autónoma de Tabasco (UJAT), México. cuauhtemoc.alvarado@ujat.mx Universidad Juárez Autónoma de Tabasco (UJAT), México. carlos.lobato@ujat.mx Universidad Juárez Autónoma de Tabasco (UJAT), México. miguel.vilchis@ujat.mx Universidad Juárez Autónoma de Tabasco (UJAT), México. Fernando.roa@ujat.mx
} 


\section{Resumo}

O escuaramide e seus derivados representam um importante grupo de substâncias que atualmente estão sendo aplicadas com sucesso em estratégias de organocatálise assimétrica, razão pela qual essa contribuição apresenta um resumo do estado da arte relacionado à síntese de derivados de escuaramida e seu papel como indutores de quiralidade em sequências sintéticas relevantes. Para tanto, foi estabelecido um processo de busca sistemática e seleção de relatórios publicados em revistas especializados da área, a fim de apresentar os aspectos mais pertinentes da síntese e a aplicação de derivados de escuaramida nesta notável área da química orgânica.

Palavras-chaves: química orgânica, síntese assimétrica, organocatalisadores, indução quiral, derivados de escuaramida.

\section{Introducción}

En este artículo de revisión se presenta una breve reseña de la historia de moléculas tipo escuaramida (estructura 1 de la figura 1), nombre sistemático es 3.4-diaminociclobut-3-en-1.2-diona, y su aplicación como organocatalizadores en síntesis asimétrica. Estas moléculas son derivados del ácido escuárico (estructura 2 de la figura 1). La síntesis del ácido escuárico fue reportada por primera vez por el grupo de investigación de Cohen (1959 citado por Thorpe, 1968).
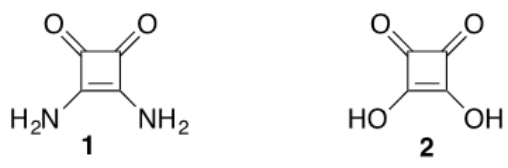

Figura 1. Escuaramida (compuesto 1) y ácido escuárico (compuesto 2).

Fuente: elaboración propia de los autores.

En 1968 Thorpe reportó la síntesis de algunos escuaramidas y los espectros de RMN-1H de estas. Desde esa fecha hubo un vacío bibliográfico hasta 1992, con el trabajo de Frauenhoff y colaboradores, el cual informó del uso de este tipo de compuestos como ligantes en química de coordinación.
A lo largo de los años, han surgido numerosas aplicaciones y estudios teóricos, debido a las inusuales propiedades fisicoquímicas de estos derivados (Ian Storer, Aciro y Jones, 2011; Quiñonero, Frontera, Ballesteros y Deyà 2000). Además, en el año 2009, resurgió el interés por este tipo de moléculas en el área de la organocatálisis. Para el mes de noviembre de 2017 se encontraron 75 artículos con la palabra clave escuaramida, siendo China y España los principales países con reportes sobre este tema. (Scopus, 2017). En este artículo de revisión, primero se abordarán los aspectos relacionados con la síntesis de escuaramidas y, luego, en un segundo apartado, se tratará el papel de estos compuestos como organocatalizadores.

\section{Síntesis de escuaramidas}

El precursor de la escuaramida como lo conocemos hoy en día es el di-cetociclobuten- diol (3.4-dihidroxiciclobut-3-en-1.2-diona), también conocido como ácido escuárico, (estructura 2 de la figura 1). Este fue preparado por primera vez en 1959 por Cohen, Lacher y Park, mediante la hidrólisis del 3.3-dicloro-1.2.4.4-tetrafluorociclobut-1-eno. (Cohen, Lacher y Park, 1959)

La mayoría de los derivados de las escuaramidas son sintetizados a partir de la 3.4-dihidroxciclobut-3-en-1.2-diona (Ian Storer et al., 2011; Kumar et al., 2012; Muthyala, Subramaniam y Todaro, 2004). A continuación, se muestran algunos ejemplos de reacciones para acceder a las escuaramidas. En 1966, Cohen y colaboradores reportaron por primera vez la síntesis de la escuaramida con rendimientos de $75 \%$ y $87 \%$ sucesivamente a partir del escuarato de dimetilo (figura 2).
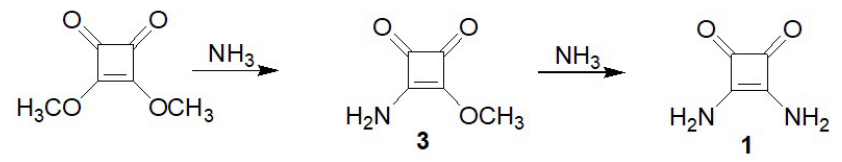

Figura 2. Síntesis de escuaramida a partir del escuarato de dimetilo.

Fuente: modificado de Cohen (1965). 
Desde ese año no se había profundizado en las aplicaciones de la escuaramida como tal, ya que inicialmente el énfasis estuvo dirigido a la comprensión estructural y la dinámica molecular del anillo de cuatro miembros de los derivados del ácido escuárico (Cohen y Cohen, 1966) debido a la geometría molecular que presentan este tipo de compuestos.

Posteriormente en el año de 1976, Eggerding y West informaron sobre una serie de derivados de mono-tioescuaratos y los iones de 1.2-ditioescuaratos y sus correspondientes derivados. En uno de los pasos de síntesis se describió la reacción de los compuestos $7 \mathrm{a}$ y $7 \mathrm{~b}$ (representados en la figura 3) con 1 equivalente de dimetilamina que provocó solamente el desplazamiento de los grupos etoxi, lo que proporcionó la serie de 3-dimetilamino-4-alquiltiociclobutenodionas (Eggerding y West, 1976) con un rendimiento de hasta un $70 \%$.
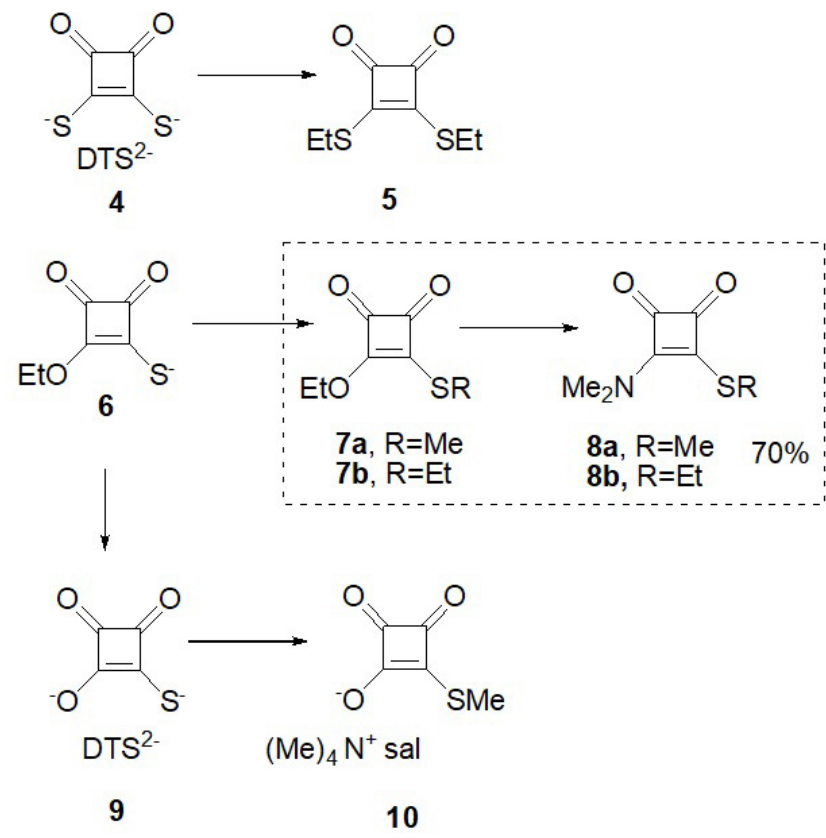

Figura 3. Síntesis de mono y tioescuaratos y derivados.

Fuente: modificado de Eggerdin y West (1976).

La aplicabilidad de las escuaramidas no era tan relevante, puesto que, como se mencionó, tuvieron que pasar alrededor de 20 años para que se realizara un nuevo estudio de este tipo de estructuras. Ya en 1995, Morley, realizó estudios teóricos sobre la estructura electrónica y propiedades no lineales de la escuaramida (Morley, 1995).

Al año siguiente, en 1996, Tomàs et al sintetizaron otra batería de derivados de escuaramida, esto con el fin de estudiar sus propiedades ácido-base y, así, poder corroborar información teórica acerca de su papel en la formación en enlaces por puentes de hidrógeno en la estabilización de complejos (figura 4). Con esto, se dio pauta a muchas aplicaciones con derivados de escuaramidas.

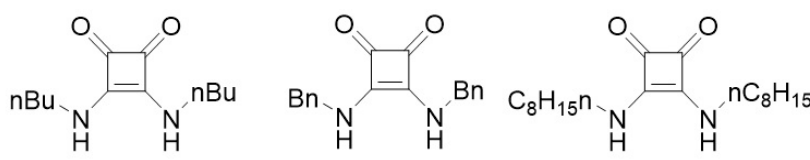

Figura 4. Derivados de escuaramida sintetizados.

Fuente: Tomàs et al. (1996).

Fue cinco años más tarde, en el 2001, que Prohens et al reportaron la síntesis de derivados de escuaramida con la finalidad de determinar sus parámetros termodinámicos (las estructuras se muestran en la figura 5). A partir de este estudio, los autores concluyeron que la capacidad para la formación de enlaces por puentes de hidrógeno puede modularse en las escuaramidas por la sustitución de grupos hidroxilo por grupos amino, además, que esta propiedad depende del tipo de solvente empleado.

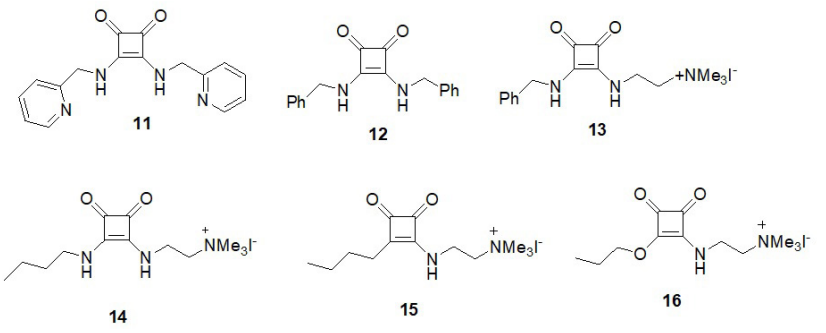

Figura 5. Batería de derivados de escuaramida.

Fuente: modificado de Prohens et al. (2001).

En el 2002, Liu, Lam, Fowler y Lauher trabajaron un derivado de escuaramida (figura 6), que prepararon fácilmente mezclando en proporciones estequiométricas escuarato de dietilo con 4-aminometilpiridina. 


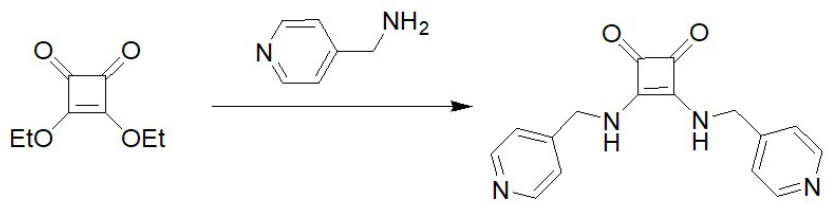

Figura 6. Síntesis de un derivado de escuaramida a partir de escuarato de dietilo y 4 -aminometilpiridina.

Fuente: modificado de Liu et al. (2002).

Estos autores encontraron que esta escuaramida tiene características muy peculiares, dado que se puede emplear una estrategia de co-cristalización para la ingeniería de cristalización. En esta, cada molécula posee una funcionalidad de enlace por puente de hidrógeno (figura 7) que conduce a la formación de una red con una distancia repetida.

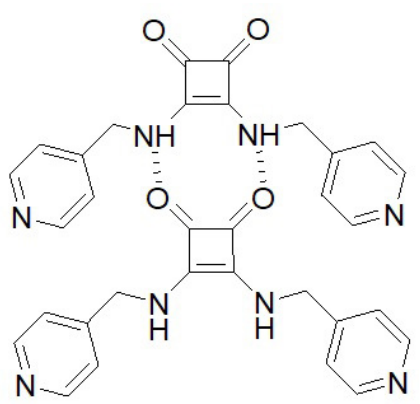

Figura 7. Interacciones por puente de hidrógeno que permite la formación de una red cristalina a partir del derivado de escuaramida.

Fuente: modificado de Liu et al. (2002).
En el 2005, Frontera y colaboradores reportaron una escuaramida para cuya síntesis se emplearon dos etapas. Como primera etapa realizaron la condensación de escuarato de dietilo y 9-(aminometil) antraceno, esto dio como resultado el intermediario el éster de escuaramida. Seguido a ello, Ilevaron a cabo una condensación con 2-(aminometil)-8-corona-6 (figura 8) para llegar al compuesto deseado con un rendimiento del 52\%.

Por otro lado, en el año 2008, Malerich, Hagihara, y Rawal reportaron un derivado de escuaramida bifuncionalizada con sustituyentes quirales (figura 9); ellos realizaron una reacción de sustitución doble para la obtención del catalizador.

\section{Las escuaramidas como organocataliza- dores}

La síntesis asimétrica consiste en la transformación de un sustrato aquiral en un producto quiral, mediante la generación de uno o más centros estereogénicos con quiralidad definida. Esta área de la química orgánica está presente en los grupos de investigación de hoy en día como una consecuencia de la tragedia de la talidomida, medicamento que fue lanzado en la década de los sesenta para el tratamiento de los síntomas del embarazo, como ansiedad, insomnio y náuseas matutinas.

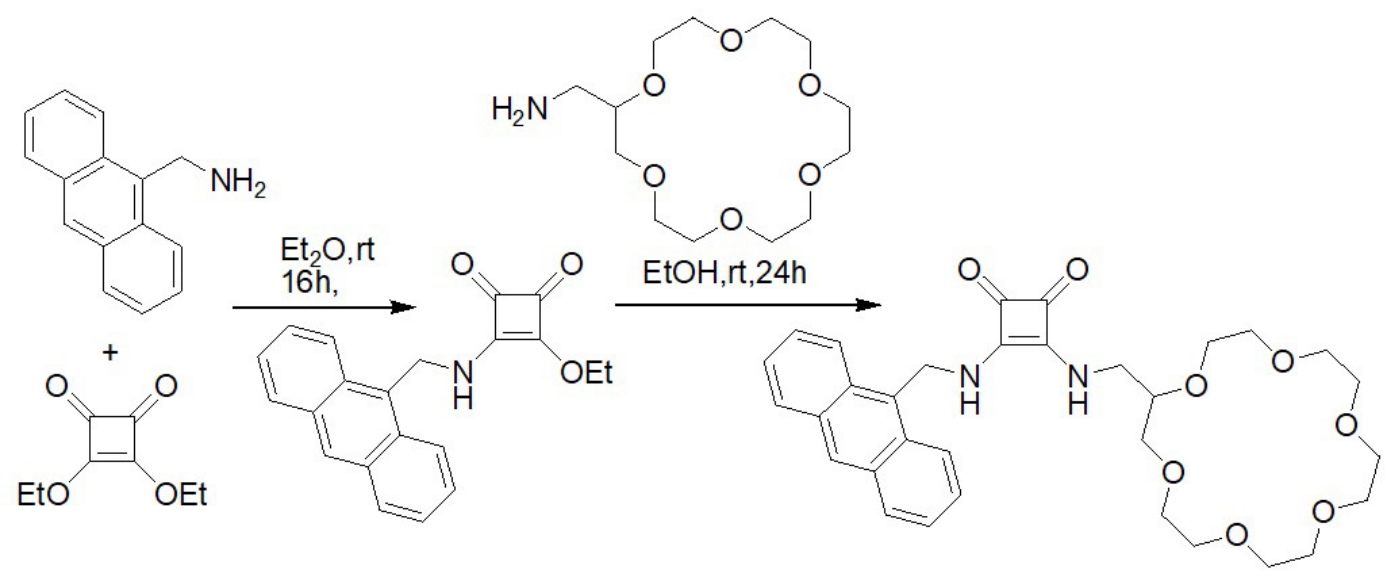

Figura 8. Síntesis de derivado de escuaramida que incluye en su estructura a un sistema de antraceno.

Fuente: modificado de Frontera et al. (2005). 

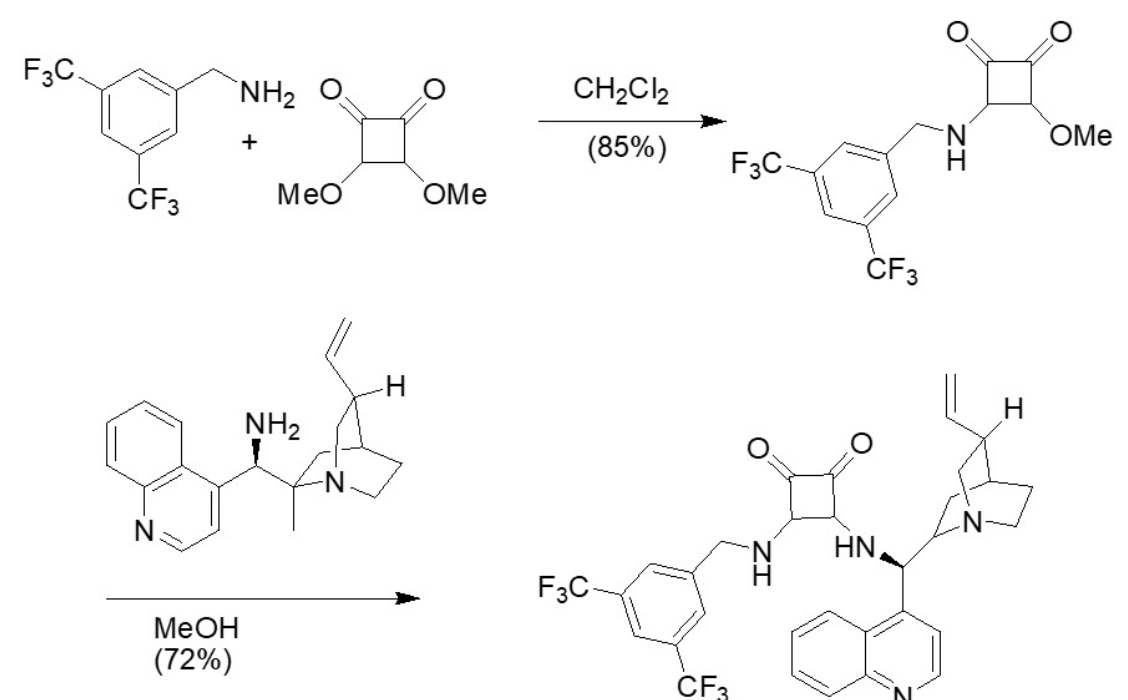

Figura 9. Proceso para la síntesis de derivado quiral de escuaramida.

Fuente: Malerich et al. (2008).

Desafortunadamente, este fue comercializado como una mezcla racémica, pero solo uno de los enantiómeros presentaba la actividad farmacológica deseada, mientras que el otro presentaba efectos teratogénicos. Esto desencadenó el nacimiento de gran número de niños con malformaciones por los efectos nocivos de esta sustancia. A raíz de ello, se enfatizó el desarrollo de procesos de síntesis y separación que llevaran a la obtención de compuestos enantioméricamente puros (Nguyen He y Pham-Huy, 2006).

Actualmente, existen diversas estrategias de síntesis que llevan a la obtención de compuestos ópticamente puros. Con este fin, en los últimos años, la organocatálisis asimétrica se ha ido posicionando cada vez más como una alternativa viable. Esto ha provocado que, en la actualidad, las escuaramidas estén siendo reconocidas como agentes potenciales para inducir quiralidad en una reacción química, y por poseer dentro de su estructura (figura 10) tanto sitios donadores como receptores para la formación de enlaces por puentes de hidrógeno ( Alemán, Parra, Jiang, y Jørgensen, 2011; Guo y Wong, 2017; Quiñonero et al, 2000).

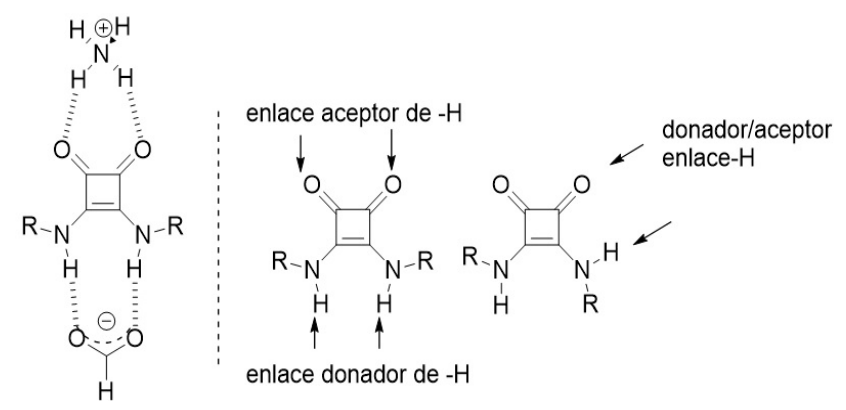

Figura 10. Dualidad en la unión bidimensional e hidrogénica de las esquaramidas.

Fuente: Alemán et al. (2011).

De esta manera, las escuaramidas se comparan en su comportamiento químico con las amidas, ureas y tioureas ya que en todas ellas se encuentran al mismo tiempo sitios moleculares con carácter donador y aceptor de enlaces por puentes de hidrógeno. (Alemán et al., 2011; Quiñonero et al., 2000). Es decir, son capaces de formar cuatro enlaces por puentes de hidrógeno (Ian Storer et al., 2011). Este gran conjunto de catalizadores asimétricos que se caracterizan por la posibilidad de generar puentes de hidrógeno para estabilizar intermediarios de reacción han sido objeto de numerosas aplicaciones, que han crecido de forma 
explosiva en la última década (Malerich et al, 2008). Aunque en el año de 1977, Wynberg y colaboradores presentaron un aporte importante en el campo de la organocatálisis, vía la formación de enlaces por puentes de hidrógeno (Guo y Wong, 2017; Helder, Arends, Bolt, Hiemstra y Wynberg., 1977).

Un aspecto a considerar en el diseño de nuevos organocatalizadores es el tipo de interacción entre éste y el sustrato, porque la activación mediante puentes de hidrógeno ha despertado el interés de diversos grupos de investigación. Se ha postulado que el papel de este tipo de catalizadores es mediante la activación no covalente que promueven los sitios del catalizador que se comportan como bases de Bröönsted al funcionar como aceptoras de protones en interacciones del tipo del puente de hidrógeno. De manera particular, los organocatalizadores del tipo escuaramida presentan claramente estos tipos de interacciones (Alemán y Cabrera, 2013; Alemán et al, 2011).

En la década pasada, ureas, tioureas, dioles y ácidos fosfóricos quirales dominaban el campo de la inducción mediante la formación de puentes de hidrógeno, (Qian et al, 2010). Sin embargo, hoy en día una serie de catalizadores a base de escuaramida se han desarrollado y aplicado con éxito en varias reacciones asimétricas (Malerich et al, 2008; Qian et al, 2010; Yang y Du, 2010) como: la reacción de Friedel-Crafts (Qian et al, 2010); la $\alpha$-aminación de compuestos 1.3-dicarbonílicos con nitroalquenos (Konishi, Lam, Malerich y Rawal, 2010) y la adición de Michael enantioselectiva (Malerich et al, 2008; Xu et al, 2010; Zhu et al, 2010).

En 2010, Qian y colaboradores demostraron que las escuaramidas quirales son catalizadores altamente eficaces para la reacción enantioselectiva de Friedel-Crafts de indoles con iminas, Qian reportó excelentes rendimientos (75-96\%) y buenos excesos enantioméricos (81-96\%).

Tabla 1. Reacción de Friedel-Crafts enantioselectiva de indoles con $\mathrm{N}$-tosiliminas catalizada por escuaramida.

\begin{tabular}{|c|c|c|c|c|c|}
\hline $\mathrm{R}_{1}$ & $\mathrm{R}_{2}$ & $\mathrm{R}_{3}$ & $\mathrm{t} / \mathrm{h}$ & Rend. $(\%)^{b}$ & $\mathrm{ee}^{\mathrm{c}}$ \\
\hline$\overline{4-\mathrm{Cl}}$ & $4-\mathrm{Me}$ & $\mathrm{H}$ & 24 & 94 & 95.5 \\
\hline $2-\mathrm{Cl}$ & 4-Me & $\mathrm{H}$ & 18 & 91 & 94 \\
\hline $4-\mathrm{Br}$ & 4-Me & $\mathrm{H}$ & 72 & 92 & 96 \\
\hline $2-\mathrm{Br}$ & 4-Me & $\mathrm{H}$ & 48 & 92 & 93 \\
\hline $4-\mathrm{F}$ & 4-Me & $\mathrm{H}$ & 72 & 90 & 92 \\
\hline $4-\mathrm{H}$ & 4-Me & $\mathrm{H}$ & 72 & 88 & 95 \\
\hline 4-Me & 4-Me & $\mathrm{H}$ & 26 & 86 & 91 \\
\hline 4-OMe & 4-Me & $\mathrm{H}$ & 48 & 89 & 93 \\
\hline 4-isopropil & 4-Me & $\mathrm{H}$ & 72 & 87 & 94 \\
\hline $4-\mathrm{H}$ & 4-Me & 5-Me & 72 & 92 & 91 \\
\hline $4-\mathrm{H}$ & 4-Me & 5-OMe & 72 & 96 & 93 \\
\hline $4-\mathrm{H}$ & $4-\mathrm{Cl}$ & $\mathrm{H}$ & 36 & 85 & 84 \\
\hline \multirow[t]{2}{*}{$4-\mathrm{H}$} & $2-\mathrm{Me}$ & $\mathrm{H}$ & 72 & 88 & 90 \\
\hline & & $\mathrm{H}$ & 92 & 75 & 81 \\
\hline
\end{tabular}

Fuente: Qian et al. (2010).

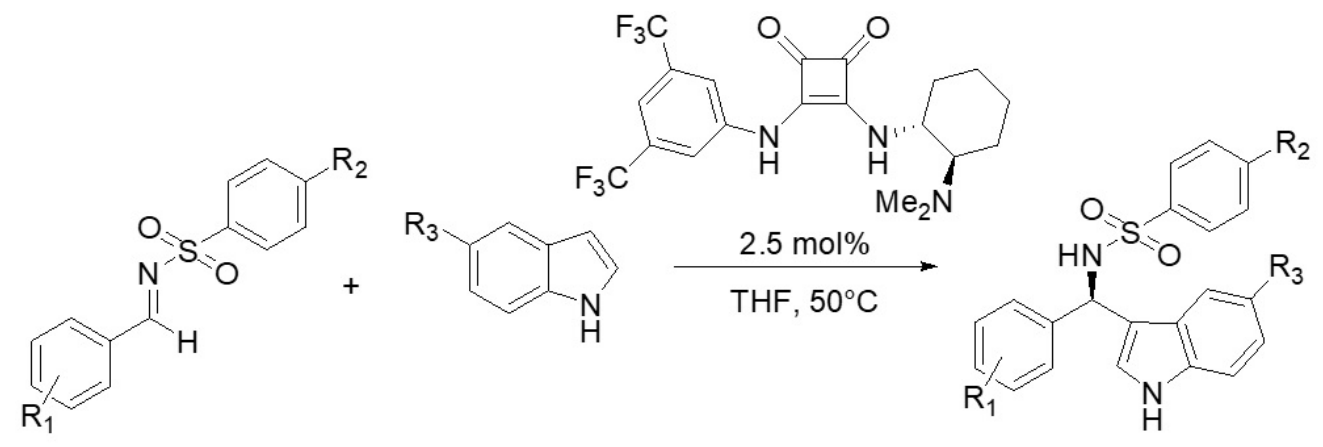

Figura 11. Reacción enantioselectiva de Friedel-Crafts de indoles con iminas.

Fuente: modificado de Qian et al. (2010). 

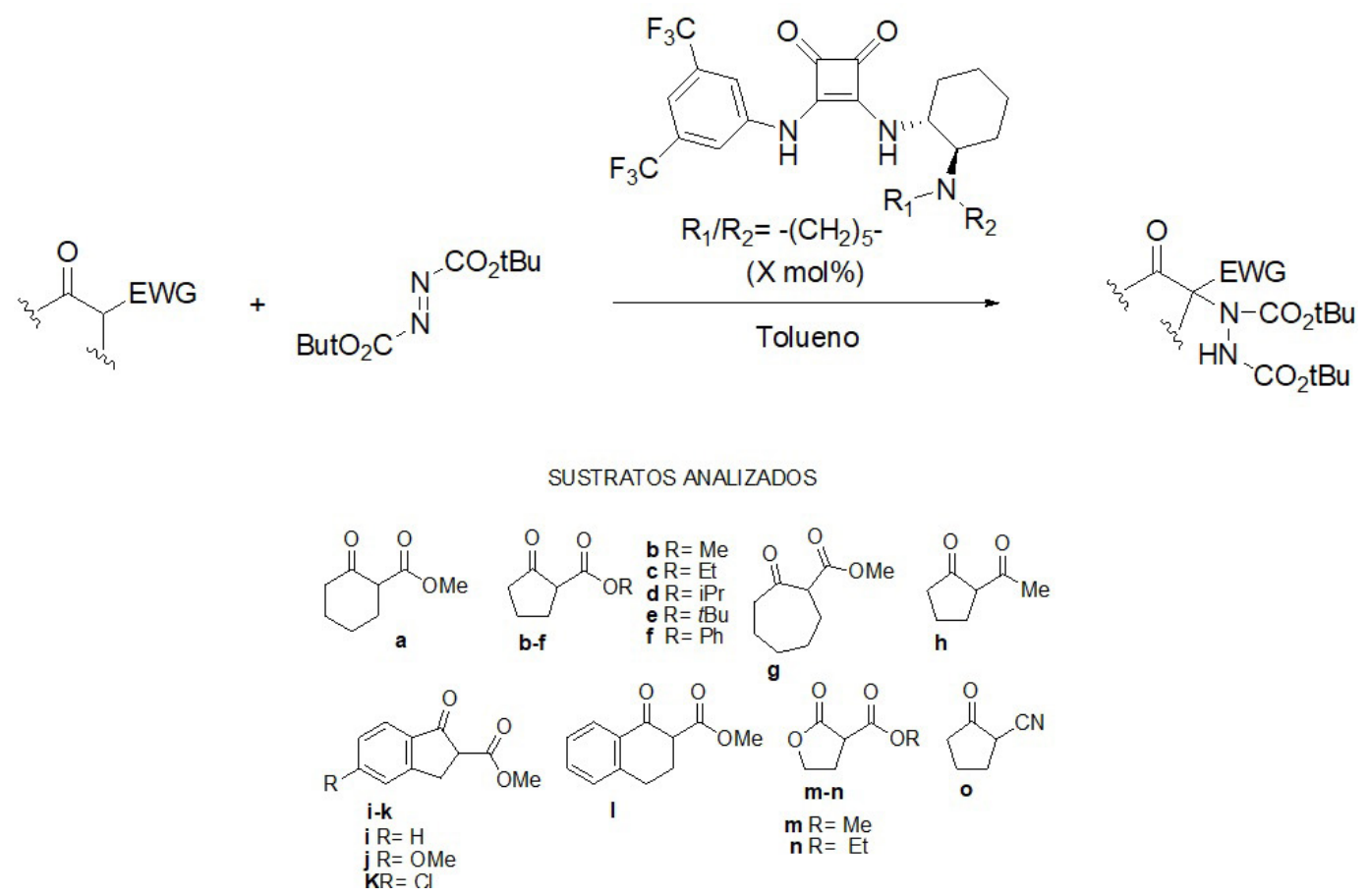

Figura 12. $\alpha$-aminación enantioselectiva de compuestos 1.3-dicarbonilicos.

Fuente: modificado de Konishi et al. (2010).

La adición de Michael asimétrica se ha convertido en una potente y eficiente herramienta para la formación de enlaces C-C en síntesis orgánica. En 2012, Kasaplar, Riente, Hartmann y Pericàs llevaron a cabo la reacción asimétrica de adición de Michael de compuestos 1.3-dicarbonílicos a $\beta$-nitroestirenos con buenos rendimientos y excelentes enantioselectividades. Utilizaron una escuaramida quiral inmovilizada covalentemente en una resina de poliestireno (tipo Merrifield), esto se muestra la estructura del catalizador en la figura 13, con el que reportaron mejores excesos enantioméricos.

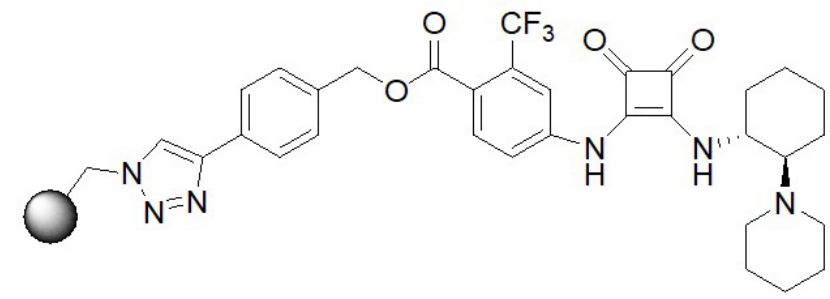

Figura 13. Catalizador derivado de escuaramida soportada en resina tipo Merrifield.

Fuente: modificado de Kasaplar et al. (2012).
Posteriormente, los autores realizaron la evaluación del alcance de la reacción con $\beta$-nitroestirenos diversamente sustituidos, y llevaron a cabo la reacción a temperatura ambiente (figura 13). Los resultados obtenidos con los compuestos probados se resumen en la tabla 2 (Kasaplar et al, 2012).

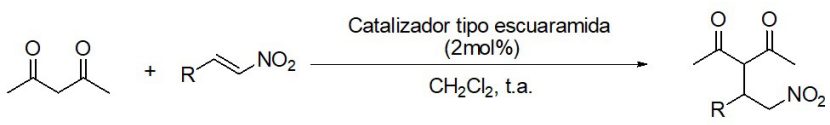

Figura 13. Adición de Michael asimétrica.

Fuente: modificado de Kasaplar et al. (2012).

En 2015, Chen et al desarrollaron una reacción altamente enantioselectiva de Michael en cascada con un proceso de ciclación empleando 3-hidroxioxindoles/3-aminooxindoles con $\alpha, \beta$-fosfonatos de acilo insaturados catalizada por derivados de escuaramida (la figura 14) Desarrollaron una amplia gama de compuestos espirocíclicos con rendimientos, que van de moderados a excelentes y de buenos a excelentes diastereo- y enantioselectividad (tabla 3). 
Tabla 2. Resultados de la adición de sistemas dicarbonílicos a nitroestirenos empleando el catalizador de escuaramida soportado en resina tipo Merrifield.

\begin{tabular}{llll}
\hline $\mathrm{R}$ & $\mathrm{t} / \mathrm{h}$ & Rend. $(\%)^{b}$ & $\mathrm{ee}^{c}$ \\
\hline $\mathrm{C}_{6} \mathrm{H}_{5}$ & 8 & 88 & 95 \\
$4-\mathrm{MeC}_{6} \mathrm{H}_{4}$ & 13 & 87 & 94 \\
$4-\mathrm{OMeC}_{6} \mathrm{H}_{4}$ & 24 & 81 & 92 \\
$2-\mathrm{OMeC}_{6} \mathrm{H}_{4}$ & 13 & 91 & 93 \\
$4-\mathrm{BrC}_{6} \mathrm{H}_{4}$ & 13 & 87 & 92 \\
$2-\mathrm{BrC}_{6} \mathrm{H}_{4}$ & 24 & 85 & 87 \\
$2.4-\mathrm{diBrC}_{6} \mathrm{H}_{3}$ & 13 & 91 & 93 \\
Furano & 13 & 91 & 94 \\
\hline${ }^{a}$ Condiciones de reacción: 0.4 mmol de 2.4-pentadiona, 0.2 mmol de nitroestireno sustituido, 2 mol\% de catalizador en 0.6 \\
ml de $\mathrm{CH}_{2} \mathrm{Cl}_{2} \cdot{ }^{b}$ Rendimiento aislado. ${ }^{c} \mathrm{El}$ ee se determinó por HPLC quiral. \\
\hline
\end{tabular}

Fuente: modificado de Kasaplar et al. (2012).

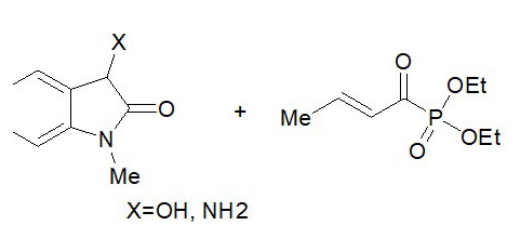

$\mathrm{X}=\mathrm{OH}, \mathrm{NH} 2$
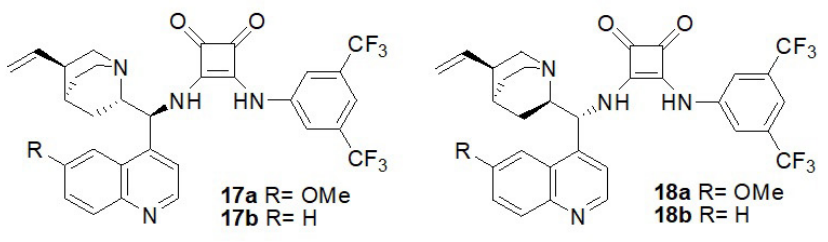

Figura 14. Ciclación de 3-hidroxioxindoles y 3-aminooxindoles con $\alpha, \beta$-fosfonatos de acilo insaturados catalizada por derivados de escuaramida.

Fuente: modificado de Chen et al. (2015).

Tabla 3. Condiciones de optimización para la obtención de espirocompuestos a partir de 3-hidroxioxindoles/3aminooxindoles con $\alpha, \beta$-fosfonatos.

\begin{tabular}{|c|c|c|c|c|c|}
\hline Cat. & Solvente & $\mathrm{t} / \mathrm{h}$ & Rend. $(\%)^{b}$ & $d r^{c}$ & ee $(\%)^{c}$ \\
\hline $17^{\mathrm{a}}$ & DCM & 9 & 53 & $>99: 1$ & $92^{d}$ \\
\hline $17 b$ & DCM & 9 & 46 & 99:1 & $93^{d}$ \\
\hline $18^{\mathrm{a}}$ & DCM & 9 & 39 & $>99: 1$ & 94 \\
\hline $18 b$ & DCM & 9 & 51 & $>99: 1$ & 94 \\
\hline $18 \mathrm{~b}$ & $\mathrm{CHCl}_{3}$ & 9 & 53 & $97: 3$ & 94 \\
\hline $18 \mathrm{~b}$ & $\mathrm{DCE}^{3}$ & 9 & 55 & $>99: 1$ & 93 \\
\hline $18 b$ & Tolueno & 6 & 38 & $94: 6$ & 85 \\
\hline $18 \mathrm{~b}$ & $\mathrm{C}_{6} \mathrm{H}_{5} \mathrm{Cl}$ & 6 & 43 & $95: 5$ & 87 \\
\hline $18 \mathrm{~b}$ & $\mathrm{THF}^{3}$ & 3 & 54 & $>99: 1$ & 94 \\
\hline $18 \mathrm{~b}$ & $\mathrm{Et}_{2} \mathrm{O}$ & 3 & 41 & $97: 3$ & 92 \\
\hline $18 b$ & $\mathrm{CH}_{3} \mathrm{CN}$ & 3 & 70 & $>99: 1$ & 94 \\
\hline $18 b$ & $\mathrm{CH}_{3} \mathrm{CN}$ & 27 & 81 & 99:1 & 94 \\
\hline $18 \mathrm{~b}$ & $\mathrm{CH}_{3} \mathrm{CN}$ & 27 & 96 & 99:1 & 94 \\
\hline
\end{tabular}

${ }^{a}$ Condiciones de reacción: hidroxioxindoles/3-aminooxindoles $(0.1 \mathrm{mmol}), \alpha, \beta$-fosfonato $(0.1 \mathrm{mmol})$ y $20 \% \mathrm{~mol}$ de catalizador (a-d) en $2.0 \mathrm{ml}$ de disolvente. ${ }^{b}$ Rendimiento aislado. ${ }^{c}$ Determinado por HPLC quiral. ${ }^{d}$ Configuración Contraria.

Fuente: Chen et al. (2015). 
De igual modo, se ha estudiado el potencial biológico de este tipo de derivados. En 2016, se reportó que tienen propiedades biológicas como antiespasmódicos e inhibidores de micobacterias (Ribeiro et al., 2016; Tantry et al., 2017), también que poseen propiedades antiinflamatorias (Busch-Petersen, 2006). En el año 2014, Olmo y colaboradores sintetizaron un derivado de escuaramida (figura 15), con resultados promisorios para el tratamiento de la enfermedad de Chagas (Olmo et al., 2014).

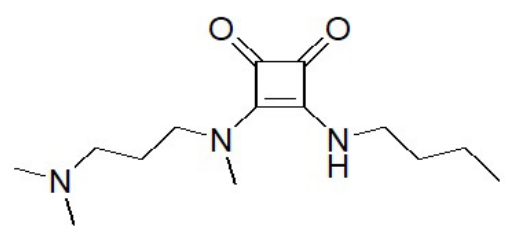

Figura 15. Derivado de escuaramida con potencial para el tratamiento de la enfermedad de Chagas.

Fuente: modificado de Olmo et al. (2014).

En el 2016, Rudd y colaboradores reportaron la síntesis de derivados del éster de escuaramida, que puede utilizarse para reacciones de bioconjugación eficaces para anticuerpos y proteínas (Rudd et al, 2016).

\section{Conclusiones}

Inicialmente, las escuaramidas fueron objeto de atención dada sus propiedades estructurales, tal como la rigidez del sistema cíclico, la relativa facilidad de interconversión en su funcionalización y las posibilidades de contar con sitios tanto aceptores como donadores en interacciones del tipo de los puentes de hidrógeno. Posteriormente, se añadieron a esta comprensión básica de un sistema estructuralmente interesante estrategias de aplicación enfocadas al uso de las escuaramidas como agentes de inducción de la quiralidad en secuencias de síntesis asimétrica, en las que se emplea como organocatalizadores quirales. Este es un ejemplo claro de cómo la contribución de un conocimiento básico sobre la estructura molecular permite el desarrollo de aplicaciones exitosas, en campos estratégicos de la ciencia. En la actualidad, se reconoce a la organocatálisis como un campo de acción de vanguardia en la síntesis asimétrica y, dentro de este campo, las escuaramidas han mostrado su eficiencia al aplicarse en nuevas reacciones enantioselectivas. Asimismo, al considerar su papel en la activación de electrófilos por la formación de enlaces tipo puente de hidrógeno.

\section{Agradecimientos}

Eduardo Hipólito Hernández agradece al Consejo Nacional de Ciencia y Tecnología (Conacyt) por la beca con número: 560217, asignada para estudios de Posgrado en programas educativos dentro del Padrón Nacional de Posgrados de Calidad (PNPC).

\section{Referencias}

Aleman, J. y Cabrera, S. (2013). Applications of asymmetric organocatalysis in medicinal chemistry. Chem Soc Rev, 42(2), 774-793. DOI: 10.1039/c2cs35380f

Alemán, J., Parra, A., Jiang, H. y Jørgensen, K. A. (2011). Squaramides: Bridging from molecular recognition to bifunctional organocatalysis. Chemistry-A European Journal, 17(25), 68906899. DOI: 10.1002/chem.201003694

Busch-Petersen, J. (2006). Small molecule antagonists of the CXCR2 and CXCR1 chemokine receptors as therapeutic agents for the treatment of inflammatory diseases. Current Topics in Medicinal Chemistry, 6(13), 1345-1352.

Chauhan, P., Mahajan, S., Kaya, U., Hack, D. y Enders, D. (2015). Bifunctional amine-squaramides: Powerful hydrogen-bonding organocatalysts for asymmetric domino/cascade reactions. Advanced Synthesis and Catalysis, 357(2-3), 253-281. DOI: 10.1002/ adsc. 201401003

Charton, J., Charruault, L., Deprez-Poulain, R. y Deprez, B. (2008). Alkylsquarates as key intermediates for the rapid preparation of 
original drug-inspired compounds. Combinatorial Chemistry and High Throughput Screening, 11(4), 294-303. DOI: $10.2174 / 138620708784246013$

Chen, L., Wu, Z.-J., Zhang, M.-L., Yue, D.-F., Zhang, X.-M., Xu, X.-Y. y Yuan, W.-C. (2015). Organocatalytic asymmetric Michael/cyclization cascade reactions of 3-hydroxyoxindoles/3-aminooxindoles with $\alpha, \beta$-unsaturated acyl phosphonates for the construction of spirocyclic oxindole- $\gamma$-lactones/lactams. The Journal of Organic Chemistry, 80(24), 1266812675. DOI: 10.1021/acs.joc.5b02253

Cohen, S., Lacher, J. R. y Park, D. (1959). Diketocyclobutenediol. Journal of the American Chemical Society, 81(13), 3480-3480. DOI: 10.1021/ja01522a083

Cohen, S. y Cohen, S. G. (1966). Preparation and reactions of derivatives of squaric acid. alkoxy-, hydroxy- and aminocyclobutenediones. Journal of the American Chemical Society, 88(7), 1533-1536. DOI: 10.1021/ja00959a040

Dai, L., Yang, H., Niu, J. y Chen, F. (2012). Enantioselective protonation in the sulfa-Michael addition using chiral squaramides as hydrogen-bonding organocatalysts. Synlett, 2, 314316. DOI: 10.1055/s-0031-1290113

Eggerding, D. y West, R. (1976). Synthesis of the monothiosquarate and 1.2-dithiosquarate ions and their derivatives. The Journal of Organic Chemistry, 41(24), 3904-3909. DOI: 10.1021/ jo00886a027

Frauenhoff, G. R., Takusagawa, F. y Busch, D. H. (1992). Transition metal complexes of the cis-dithiosquaramide ligand family. Inorganic Chemistry, 31, 4002-4007.

Frontera, A., Orell, M., Garau, C., Quiñonero, D., Molins, E., Mata, I. y Morey, J. (2005). Preparation, solid-state characterization and computational study of a crown ether attached to a squaramide. Org Lett, 7(8), 1437-1440. DOI: 10.1021/ol0474608

Guo, J. y Wong, M. W. (2017). Cinchona alkaloid-squaramide catalyzed sulfa-Michael addition reaction: mode of bifunctional activation and origin of stereoinduction. The Journal of Organic Chemistry, 82(8), 4362-4368. DOI: 10.1021/acs.joc.7b00388

Helder, R., Arends, R., Bolt, W., Hiemstra, H. y Wynberg, H. (1977). Alkaloid catalyzed asymmetric synthesis III: the addition of mercaptans to 2-cyclohexene-1-one; determination of enantiomeric excess using ${ }^{13} \mathrm{C}$ NMR. Tetrahedron Letters, 18(25), 2181-2182.

Ian Storer, R., Aciro, C. y Jones, L. H. (2011). Squaramides: physical properties, synthesis and applications. Chemical Society Reviews, 40(5), 2330-2346. DOI: 10.1039/COCS00200C

Kasaplar, P., Riente, P., Hartmann, C. y Pericàs, M. A. (2012). A polystyrene-supported, highly recyclable squaramide organocatalyst for the enantioselective Michael addition of 1.3-dicarbonyl compounds to $\beta$-nitrostyrenes. Advanced Synthesis \& Catalysis, 354(16), 2905-2910. DOI: 10.1002/ adsc. 201200526

Konishi, H., Lam, T. Y., Malerich, J. P. y Rawal, V. $H$. (2010). Enantioselective $\alpha$-amination of 1.3-dicarbonyl compounds using squaramide derivatives as hydrogen bonding catalysts. Organic Letters, 12(9), 2028-2031. DOI: 10.1021/ ol1005104

Kumar, S. P., Gloria, P. M. C., Goncalves, L. M., Gut, J., Rosenthal, P. J., Moreira, R. y Santos, M. M. M. (2012). Squaric acid: a valuable scaffold for developing antimalarials? MedChemComm, 3(4), 489-493. DOI: 10.1039/ C2MD20011B

Liu, Y., Lam, A. H. W., Fowler, F. W. y Lauher, J. W. (2002). The squaramides. A new family of host molecules for crystal engineering. Molecular Crystals and Liquid Crystals, 389(1), 3946. DOI:10.1080/713738914

Malerich, J. P., Hagihara, K., y Rawal, V. H. (2008). Chiral squaramide derivatives are excellent hydrogen bond donor catalysts. Journal of the American Chemical Society, 130(44), 1441614417. DOI: 10.1021/ja805693p 
McCooey, S. H. y Connon, S. J. (2005). Urea- and thiourea-substituted cinchona alkaloid derivatives as highly efficient bifunctional organocatalysts for the asymmetric addition of malonate to nitroalkenes: inversion of configuration at C9 dramatically improves catalyst performance. Angewandte Chemie International Edition, 44(39), 6367-6370. DOI: 10.1002/ anie. 200501721

Morley, J. O. (1995). Theoretical studies on the electronic structure and nonlinear properties of dicyanomethylene substituted squaramides, croconamides and rhodizonamides. Journal of Molecular Structure: THEOCHEM, 357(1), 4957. DOI: 10.1016/0166-1280(95)04279-F

Muthyala, R. S., Subramaniam, G. y Todaro, L. (2004). The use of squaric acid as a scaffold for cofacial phenyl rings. Org. Lett., 6(25), 46634665. DOI: $10.1021 / \mathrm{ol048139s}$

Nguyen, L. A., He, H. y Pham-Huy, Ch. (2006). Chiral drugs: an overview. Int. J. Biomed. Sci., 2(2), 85-100.

Olmo, F., Rotger, C., Ramirez-Macias, I., Martinez, L., Marin, C., Carreras, L., Costa, A. (2014). Synthesis and biological evaluation of $\mathrm{N}, \mathrm{N}^{\prime}$-squaramides with high in vivo efficacy and low toxicity: toward a low-cost drug against Chagas disease. J. Med. Chem., 57(3), 987-999. DOI: 10.1021/jm4017015

Prohens, R., Rotger, M. C., Piña, M. N., Deyà, P. M., Morey, J., Ballester, P. y Costa, A. (2001). Thermodynamic characterization of the squaramide-carboxylate interaction in squaramide receptors. Tetrahedron Letters, 42(29), 4933-4936.

Qian, Y., Ma, G., Lv, A., Zhu, H.-L., Zhao, J. y Rawal, V. H. (2010). Squaramide-catalyzed enantioselective Friedel-Crafts reaction of indoles with imines. Chemical Communications, 46(17), 3004-3006. DOI: 10.1039/ B922120D

Quiñonero, D., Frontera, A., Ballester, P. y Deyà, P. M. (2000). A theoretical study of aromaticity in squaramide and oxocarbons. Tetrahedron
Letters, 41(12), 2001-2005. DOI: 10.1016/ S0040-4039(00)00084-8

Ribeiro, C. J. A., Espadinha, M., Machado, M., Gut, J., Gonçalves, L. M., Rosenthal, P. J. y Santos, M. M. M. (2016). Novel squaramides with in vitro liver stage antiplasmodial activity. Bioorganic \& Medicinal Chemistry, 24(8), 1786-1792. DOI: 10.1016/j.bmc.2016.03.005

Rudd, S. E., Roselt, P., Cullinane, C., Hicks, R. J. y Donnelly, P. S. (2016). A desferrioxamine B squaramide ester for the incorporation of zirconium-89 into antibodies. Chemical Communications, 52(80), 11889-11892. DOI: 10.1039/ C6CC05961A

Tantry, S. J., Markad, S. D., Shinde, V., Bhat, J., Balakrishnan, G., Gupta, A. K. y Hameed, P. S. (2017). Discovery of imidazo[1.2-a]pyridine ethers and squaramides as selective and potent inhibitors of mycobacterial Adenosine Triphosphate (ATP) synthesis. J. Med. Chem., 60(4), 1379-1399. DOI: 10.1021/acs. jmedchem.6b01358

Tomàs, S., Prohens, R., Vega, M., Rotger, M. C., Deyà, P. M., Ballester, P. y Costa, A. (1996). Squaramido-based receptors: design, synthesis, and application to the recognition of tetraalkylammonium compounds. The Journal of Organic Chemistry, 61(26), 9394-9401. DOI: 10.1021/jo9614147

Thorpe, J. E. (1968). ' H Nuclear Magnetic Resonance spectra of some squaramides. Journal of the Chemical Society B: Physical Organic, 435-436.

Rouf, A. y Tanyeli, C. (2016). Squaramide based organocatalysts in organic transformations. $\mathrm{Cu}$ rrent Organic Chemistry, 20(28), 2996-3013. DOI: $10.2174 / 138527282066616080511374$ 9

Scopus (2017). Análisis de resultados para el término "squaramide". Recuperado de https://bit. Iy/2jOrVDA

Sonsona, I.G., Marqués-López, E., Herrera, R.P. (2015). Enantioselective organocatalyzed synthesis of 2-amino-3-cyano-4H-chromene 
derivatives. Symmetry, 7(3), 1519-1535. DOI: 10.3390/sym7031519

Sonsona, I. G., Marqués-López, E. y Herrera, R. P. (2016). The aminoindanol core as a key scaffold in bifunctional organocatalysts. Beilstein Journal of Organic Chemistry, 12, 505-523. DOI: 10.3762/bjoc. 12.50

Storer, R. I., Aciro, C. y Jones, L. H. (2011). Squaramides: physical properties, synthesis and applications. Chemical Society Reviews, 40(5), 2330-2346.

Vetica, F., De Figueiredo, R.M., Orsini, M., Tofani, D. y Gasperi, T. (2015). Recent advances in organocatalytic cascade reactions toward the formation of quaternary stereocenters. Synthesis, 47(15), 2139-2184. DOI: 10.1055/s-0034-1378742

Wurm, F. R. y Klok, H. A. (2013). Be squared: expanding the horizon of squaric acid-mediated conjugations. Chemical Society Reviews, 42(21), 8220-8236. DOI: 10.1039/ c3cs60153f

Xu, D.-Q., Wang, Y.-F., Zhang, W., Luo, S.-P., Zhong, A.-G., Xia, A.-B. y Xu, Z.-Y. (2010). Chiral squaramides as highly enantioselective catalysts for Michael addition reactions of 4-hydroxycoumarins and 4-hydroxypyrone to $\beta, \gamma$-unsaturated $\alpha$-keto esters. Chemistry - $A$ European Journal, 16(14), 4177-4180. DOI: 10.1002/chem.201000094

Yang, W. y Du, D.-M. (2010). Highly enantioselective Michael addition of nitroalkanes to chalcones using chiral squaramides as hydrogen bonding organocatalysts. Organic Letters, 12(23), 5450-5453. DOI: 10.1021/ol102294g

Žabka, M. y Šebesta, R. (2015). Experimental and theoretical studies in hydrogen-bonding organocatalysis. Molecules, 20(9), 15500-15524. DOI: 10.3390/molecules 200915500

Zhang, J., Zhou, H.-B. y Xie, R.-G. (2002). $\mathrm{N}$-substituted amides as chiral ligands for catalytic asymmetric reactions. Current Organic Chemistry, 6(10), 865-890. DOI: 10.2174/1385272023373806

Zhao, B. L., Li, J. H. y Du, D. M. (2017). Squaramide-catalyzed asymmetric reactions. Chemical Record, 17(10), 994-1018. DOI: 10.1002/ tcr.201600140

Zhu, Y., Malerich, J. P. y Rawal, V. H. (2010). Squaramide-catalyzed enantioselective Michael addition of diphenyl phosphite to nitroalkenes. Angewandte Chemie International Edition, 49(1), 153-156. DOI: 10.1002/ anie.200904779 